\title{
Role of ARHGAP42 in hypertension
}

expression of
[ARHGAP42]
might act as
a negative
feedback
mechanism to
limit excessive
vessel
constriction

פ
A new study suggests a role of the ARHGAP42 genotype in determining risk of hypertension. Joan Taylor, Christopher Mack and colleagues previously identified ARHGAP42 as a Rho GTPase-activating protein that is selectively expressed in smooth muscle cells (SMCs) and controls blood pressure (BP) by regulating vascular tone.

The researchers have now identified a regulatory element in ARHGAP42 that encompasses the BP-associated single nucleotide polymorphism, rs604723, and has SMCselective activity. CRISPR/Cas9-mediated deletion of this regulatory element significantly reduced endogenous ARHGAP42 expression in human aortic SMCs. Moreover, the presence of the minor T allele at rs604723 enhanced the activity of the regulatory element. "The presence of this allele created a low-affinity binding site for serum response factor, which has previously been implicated in SMC-specific gene expression," says Mack.

ARHGAP42 expression in vascular SMCs was upregulated by cell stretch, hypertension, and RhoA-dependent agonists, suggesting that expression of this GTPase might act as a negative feedback mechanism to limit excessive vessel constriction. Consistent with this hypothesis, Arhgap42deficient mice showed increased susceptibility to DOCA-salt-mediated hypertension.

Finally, the researchers showed that the minor $\mathrm{T}$ allele of ARHGAP42 was associated with reduced diastolic BP in patients with untreated borderline hypertension. "Genotypic analysis of approximately 1,000 individuals from several additional clinical cohorts suggested that the low frequency of the minor ARHGAP42 allele in African Americans might contribute to the susceptibility of this population to the development of hypertension," comments Taylor. "Our findings could perhaps lead to the development of personalized treatment options for hypertension based on ARHGAP42 genotype."

Ellen F. Carney

ORIGINAL ARTICLE Bai, X. et al. Blood-pressure-associated polymorphism controls ARHGAP42 expression via serum response factor DNA binding.J. Clin. Invest. http://dx.doi. org/10.1172/JCl88899 (2017) 